Introduction Brushings at ERCP play a crucial role in establishing a diagnosis in cases of biliary strictures. In this study, we examined if brushing practice can make a difference to the diagnostic yield resulting in less interventions. We analysed ERCP data over the last two and a half years at the Royal Berkshire Hospital, where inclusion of the brush head for cytology and performing three or more passages across the stricture has been standard practice.

Methods We looked at results of brushings taken by 2 ERCPists in a single centre between September 2016 to February 2018. The initial brushing result from each individual patient was included. Brushing results were classified as non-diagnostic, negative, atypical, suspicious or positive for malignancy. Brushings classified as suspicious and identified as malignant were categorised into the positive group; those classified as non-diagnositc, non-malignant and atypical were categorised as negative. For comparison purposes we considered the corresponding histology and/or radiological findings and/or positive immunohistochemistry in the follow-up period. Data was analysed using the statistical package SPSS v.25.

Results In total, 71 individual brushing outcomes were identified over a 2.5-year period. In 61 of 71 cases $(85.9 \%)$ the final diagnosis was cancer, with the majority being pancreatic cancer $(36 / 61 ; 59 \%)$. The sensitivity was estimated at $77 \%$ (47/61 true positive), the specificity and the positive predictive value (PPV) were both $100 \% \quad(0$ false negative, 10 true negative), while the negative predictive value (NPV) was $38 \%$. When we looked at only 'positive for malignancy' results, the sensitivity stood at $60 \%$. In $15 / 61$ cases $(24.6 \%)$ a supplementary report was needed to confirm the result and it changed the outcome in only 3 cases (20\%).

Conclusions Biliary brushings are a very useful means of providing a diagnosis during ERCP and are characterised by a high PPV and low NPV. Negative results should not be interpreted as absence of malignancy but if the yield is at least suspicious, the specificity approaches 100\%. This study suggests that sending the brush head for cytology and performing three or more passages across the stricture increases diagnostic sensitivity when compared to published data reporting on sensitivities up to 64\% (Burnett AS, Calvert TJ, Chokshi RJ. Sensitivity of endoscopic retrograde cholangiopancreatography standard cytology: 10-y review of the literature. J Surg Res. 2013;184:304-11.)

\section{PTU-125 FACTORS INFLUENCING INCREASED ERCP BRUSHING SENSITIVIY IN PANCREATICOBILIARY MALIGNANCY: A SINGLE CENTRE EXPERIENCE}

loannis Varmpompitis*, Cameron Griffiths, Sinead McEvoy, Jonathan Booth, Nishchay Chandra. Royal Berkshire Hospital, Reading, UK

\subsection{6/gutjnl-2019-BSGAbstracts.114}

Introduction Biliary brushings are often the only way to confirm a diagnosis of malignancy in patients presenting with biliary strictures. There is a paucity of data regarding the parameters that may affect the diagnostic yield of this technique. The aim of this study was to identify key factors that may improve diagnostic sensitivity in hepatobiliary malignancies.

Methods Brushing outcomes were identified over a 2-year period and analysed by demographic factors (age, sex), indication of the procedure, site and length of stenosis on ERCP, CBD dilatation on CT/MRCP/ERCP, site and size of mass, laboratory values (ALP, ALT, bilirubin) prior to the procedure, and final outcome (histologically or radiologically confirmed). We calculated the ratio of at least suspicious for malignancy results out of the total cases of malignancies. Final outcome was defined by histology or radiological evidence of cancer in the follow-up period. Data was retrospectively retrieved and processed using the EPR (electronic patient record) hospital database and the radiology InSight PACS system. Data was analysed using the statistical package SPSS v.25.

Results A total of 59 brushing results were identified over the period 01/2017-01/2019. $52(88.1 \%)$ were malignant and 7 (11.9\%) were benign. The mean age was 71.4 yrs and the majority of malignancies were pancreatic cancer (33/52; $63.5 \%$ ), with the remainder including cholangiocarcinoma (7), ampullary cancer (6) and other malignancies. Forty of 52 cases were true positives, indicating a sensitivity of $76.9 \%$, while the specificity was $100 \%$ (no false negative and $7 / 7$ true negative).

Among the factors examined, sensitivity was significantly associated with the site of stenosis $(89.2 \%$ for distal vs. $50 \%$ for mid and mid-distal stenoses, $\mathrm{p}=0.023$ ), and with the mass being in the pancreatic head versus in the uncinate process (84.6 vs $40 \%, p=0.029)$. Sensitivity was higher for longer strictures $(63.6 \%$ vs $46 \%$ for strictures $>1.9 \mathrm{~cm}$ (i.e. the median value), and for larger masses (mean size of mass $4 \mathrm{~cm}$ in true positive vs $2.6 \mathrm{~cm}$ in false negatives) but these results did not reach statistical significance. The age, CBD dilatation on imaging, type of cancer and the laboratory markers before the procedure were not associated with differences in the diagnostic yield.

Conclusions Distal CBD stenoses and pancreatic head lesions are associated with statistically significant increase in brushing sensitivity for malignancy. This study suggests that in the absence of a distal CBD stricture or a pancreatic head lesion, further investigation modalities (additional brushings, EUS, percutaneous biopsy, cholangioscopy) will be more likely required to achieve diagnostic certainty.

\section{PTU-126 RESULTS OF USING COLORECTAL SEMS VS DECOMPRESSION TUBE AS BRIDGE TO SURGERY: COMPARATIVE RANDOMIZED TRAIL}

${ }^{1,2,3}$ Alexandr Vodoleev*, ${ }^{1}$ Vladimir Duvanskiy, ${ }^{2}$ Sergey Pirogov, ${ }^{3}$ Denis Kryazhev, ${ }^{2}$ lliya Perfilev, ${ }^{2}$ Dmitriy Sukhin, ${ }^{2}$ Elena Karpova, ${ }^{2}$ Andrey Kniazev, ${ }^{1}$ Ivan Yarotskov. ${ }^{1} R U D N$, Moscow, Russian Federation; ${ }^{2}$ National Medical Research Center of Radiology, Moscow, Russia; ${ }^{3}$ Eramishantsev Clinical Hospital, Moscow, Russia

\subsection{6/gutjnl-2019-BSGAbstracts. 115}

Introduction Despite advances in screening and early diagnosis, about $15 \%$ of colorectal cancers present with acute colonic 
obstruction. Emergency colorectal surgery is associated with high morbidity and mortality rates.

Preoperative decompression (SEMS or trananal decompression tube placement) as a bridge to surgery has been reported to improve short-term outcomes. However, oncological safety of endoscopic interventions is debatable due to the high frequency of perforations and dissemination. There are few retrospective studies of comparative outcomes between SEMS and decompression tube, but the benefits were not clear. The aim of this prospective randomized study is to compare sortterm and especially long term outcome of colorectal stenting and transanal decompression tube as a bridge to surgery.

Methods Between December 2012 and December 2017, 72 patients with malignant colon obstruction, which was diagnosed based on radiological and endoscopic findings with pathohistological examination, were consecutively included into the study. Patients were randomized of random sampling numbers and divided into the stent or decompression tube group.

Results Technical success rate RR 0.914, 95\% CI 0.804-1.039 $(p=0.065)$. Clinical success was significant higher in stent group RR 0.643 , 95\% CI $0.489-0.846(\mathrm{p}=0.031)$. Rate of complications was similar in groups RR 0.895 , 95\% CI $0.755-1.061 \quad(p=0.087)$. Short-term outcomes in SEMS group were superior to decompression tube group in the following areas: stoma creation rate RR 0.914 , 95\% CI $0.804-1.039$ $(p=0.065) \quad(p<0.01)$, need to ICU RR $0.673,95 \%$ CI $0.493-$ $0.919(\mathrm{p}<0.05)$, surgical infection rate RR 0.721 , 95\% CI $0.520-0.999(p<0.05)$. No significant differences were found in the overall postoperative complication rate $\mathrm{RR} 0.748,95 \%$ CI $0.535-1.044(\mathrm{p}=0.117)$, anastomotic leak rate RR 0.953, $95 \%$ CI $0.828-1.056(\mathrm{p}=0.062)$, duration of in-patient stay $17(10-21)$ and $20(14-25)$ days $(p=0.248)$. In long-term period rate of recurrence was significant higher in stent group $29.4 \%$ vs $17.8 \%(\mathrm{p}=0.031)$. However, the1-year overall survival rate $89.0 \%$ and $83.3 \%(\mathrm{p}=0.423)$, and 3 -year of patients $69.4 \%$ and $63.9 \%(\mathrm{p}=0.460)$, in the stent and tube groups, respectively, was not significant different.

Conclusions SEMS has some benefits in comparison with decompression tubes in the short-term postoperative period for patients with malignant colorectal obstruction. The overall recurrence rate was higher in the stent group in long-term period. Moreover, the overall survival rate in the group is no different. Need to continue research to material accumulation and analysis of long-term results.

\section{PTU-127 POOR QUALITY OF CAPSULE ENDOSCOPY IMAGES HAS NEGATIVE EFFECT ON DIAGNOSIS OF SMALL BOWEL MALIGNANCY}

Diana Yung*, Anastasios Koulaouzidis, John Plevris. The Royal Infirmary of Edinburgh, Edinburgh, UK

\subsection{6/gutjnl-2019-BSGAbstracts.116}

Introduction Capsule endoscopy (CE) is the prime mode of investigation for small bowel (SB) pathology. It relies heavily on image quality, which is commonly affected by poor preparation. Currently, there is no widely-accepted method for quantifying visualisation quality. We studied the contribution of various image parameters to visualisation quality and their effect on certainty of diagnosis of small bowel lesions.
Methods Five clear CE images of common SB pathology were processed for 3 parameters to simulate increasingly poor SB preparation: opacity (colour-matched to luminal content; 10 90\%, 10\% increments), blurriness (radius 1-10px; 1px increments), contrast $(-50-50 \% ; 10 \%$ increments). 9 expert readers evaluated whether images were adequate for diagnostic purposes. The points where perception of image quality changed significantly were determined for each parameter. Three further sets of SBCE images (vascular, inflammatory and malignant lesions) were processed for 4 points/parameter. 20 experienced-expert CE readers from reviewed the resulting images.

Results In vascular and inflammatory lesions, diagnostic certainty was least affected by increasing image opacity, requiring opacities $>90 \%$ before most readers considered images inadequate for diagnosis. The greatest negative effects of image opacity were seen in malignancy where significantly fewer readers found images adequate at $>50 \%$ opacity. Similar results obtained with increasing blur radius, simulating motion blur and poor focus. The proportions of readers finding vascular and inflammatory images adequate for diagnosis did not drop significantly at wider blur radii, while the proportion who found images of malignancies diagnostically adequate dropped at blur radius $6 \mathrm{px}$. Decreasing contrast had greater negative effect than raised contrast, most obvious in malignant lesions.

Conclusions Poor visualisation quality in all parameters had the greatest effect on malignant lesions. Software to increase contrast and sharpen images can improve visualisation quality; smart frame rate adaptation could also improve the number of high-quality frames obtained. Furthermore, our results suggest that thoroughness in SB cleansing is most important when there is suspicion of SB malignancy, to improve diagnostic certainty of images obtained.

\section{PTU-128 DOUBLE-HEADED CAPSULE ENDOSCOPY: REAL-WORLD EXPERIENCE FROM A MULTICENTRE BRITISH STUDY}

${ }^{1}$ Diana Yung*, 'Joanna Brzeszczynska, ${ }^{2}$ Imdadur Rahman, ${ }^{3}$ Leena Sinha, ${ }^{4}$ Reena Sidhu, ${ }^{2}$ Praful Patel, ${ }^{3}$ Sue Mason, ${ }^{4}$ Mark McAlindon, ${ }^{1} J o h n$ Plevris, ${ }^{1}$ Anastasios Koulaouzidis. ${ }^{1}$ The Royal Infirmary of Edinburgh, Edinburgh, UK; ' 2 Southampton General Hospital, Southampton, UK; ${ }^{3}$ Queen's Hospital, Romford, London, UK; ${ }^{4}$ Royal Hallamshire Hospital, Sheffield, UK

\subsection{6/gutjnl-2019-BSGAbstracts.117}

Introduction Capsule endoscopy (CE) is a well-established mode of investigation for small bowel (SB) pathology. This study examines the potential benefits of using double-headed capsules compared to conventional single-headed ones in a real-world cohort of patients referred for CE. We present initial results from the first multicentre British study.

Methods Over a 9-month period, patients referred for routine SBCE at 4 tertiary referral centres in the UK underwent double-headed CE in lieu of conventional single-headed CE using MiroCam MC2000 capsules. CE was carried out as per routine protocols at each centre. Clinical data were anonymised. One head $(\mathrm{L} / \mathrm{R})$ was chosen at random and reported by an expert reviewer. The double-headed recordings, also anonymised and randomised, were reported by another expert reviewer. In centres with only one expert reviewer, doubleheaded CEs were read after a 4-week interval to minimise recall bias. For each CE, numbers and types of findings and 